\title{
Concordance of Time-of-Flight MRA and Digital Subtraction Angiography in Adult Primary Central Nervous System Vasculitis
}

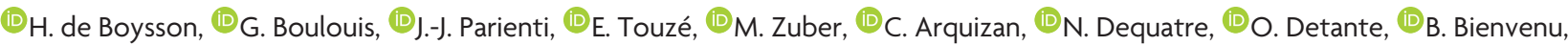 \\ (D) A. Aouba, (1) L. Guillevin, (D) C. Pagnoux, and (D) O. Naggara
}

수을

\begin{abstract}
BACKGROUND AND PURPOSE: 3D-TOF-MRA and DSA are 2 available tools to demonstrate neurovascular involvement in primary central nervous system vasculitis. We aimed to compare the diagnostic concordance of vessel imaging using 3D-TOF-MRA and DSA in patients with primary central nervous system vasculitis.
\end{abstract}

MATERIALS AND METHODS: We retrospectively identified all patients included in the French primary central nervous system vasculitis cohort of 85 patients who underwent, at baseline, both intracranial 3D-TOF-MRA and DSA in an interval of no more than 2 weeks and before treatment initiation. Two neuroradiologists independently reviewed all 3D-TOF-MRA and DSA imaging. Brain vasculature was divided into 25 arterial segments. Concordance between 3D-TOF-MRA and DSA for the identification of arterial stenosis was assessed by the Cohen $\kappa$ Index.

RESULTS: Thirty-one patients met the inclusion criteria, including 20 imaged with a $1.5 T$ MR unit and 11 with a 3 T MR unit. Among the 25 patients (81\%) with abnormal DSA findings, 24 demonstrated abnormal 3D-TOF-MRA findings, whereas all 6 remaining patients with normal DSA findings had normal 3D-TOF-MRA findings. In the per-segment analysis, concordance between 1.5T 3D-TOF-MRA and DSA was 0.82 (95\% Cl, 0.75-0.93), and between 3T 3D-TOF-MRA and DSA, it was 0.87 (95\% Cl, 0.78-0.91).

CONCLUSIONS: 3D-TOF-MRA shows a high concordance with DSA in diagnostic performance when analyzing brain vasculature in patients with primary central nervous system vasculitis. In patients with negative 3T 3D-TOF-MRA findings, the added diagnostic value of DSA is limited.

ABBREVIATIONS: COVAC = COhort of patients with primary VAsculitis of the Central nervous system; PCNSV = primary central nervous system vasculitis; $\mathrm{RCVS}=$ reversible cerebral vasoconstriction syndrome; $\mathrm{SCA}=$ superior cerebellar artery

$\mathrm{T}$ he diagnosis of primary central nervous system vasculitis (PCNSV) relies on the evidence of isolated vascular involvement of CNS vessels in patients with persistent neurologic disorders. Complete work-up should exclude other conditions that

\section{Received March 16, 2017; accepted after revision May 20}

From the Departments of Internal Medicine (H.d.B., B.B., A.A.) and Neurology (E.T.) Caen University Hospital, University of Caen-Normandie, Institut National de la Santé et de la Recherche Médicale Unite Mixte de Recherche 919, Caen, France; Department of Neuroradiology (G.B., O.N.), Centre Hospitalier Sainte-Anne, Université Paris-Descartes, Institut National de la Santé et de la Recherche Médicale Unite Mixte de Recherche 894, Paris, France; Biostatistics and Clinical Research Unit (J.-J.P.), Caen University Hospital, Caen, France; Department of Vascular Neurology (M.Z.), Centre Hospitalier Saint Joseph, Université Paris-Descartes, Institut National de la Santé et de la Recherche Médicale Unite Mixte de Recherche S 919, Paris, France; Department of Neurology (C.A.), Montpellier University Hospital, Institut National de la Santé et de la Recherche Médicale Unite Mixte de Recherche 894, Montpellier, France; Department of Neurology (N.D.), Lille University Hospital, Lille, France; Department of Neurology (O.D.), Grenoble Alpes University Hospital, Grenoble, France; Department of Internal Medicine (L.G.), Centre Hospitalier Cochin, Paris, France; and Vasculitis Clinic (C.P.), Division of Rheumatology, Mount Sinai Hospital, Toronto, Ontario, Canada.

This study was supported by an institutional grant from the French Ministry of Health (COVAC, 2009 PHRC 08017). may explain vascular changes, such as infection, malignancy, autoimmune disease, and other cerebral vasculopathies, especially reversible cerebral vasoconstriction syndrome (RCVS) or atherosclerosis. ${ }^{1-4}$ Original diagnostic criteria from Calabrese and Mallek, ${ }^{5}$ established in 1988, proposed that vascular involvement could be demonstrated with brain biopsy, which remains the criterion standard, or with DSA. DSA has a high sensitivity for the involvement of small vessels of up to $500 \mu \mathrm{m}$. However, it remains an invasive tool with the risk of rare complications. ${ }^{6,7}$

With the development and gradual improvement of MR imaging technologies, alternative options to image CNS vessels have become available and may be substitutes for DSA. MR imaging is now considered a requirement in all patients with a suspected

\footnotetext{
Please address correspondence to Hubert de Boysson, MD, MSc, Department of Internal Medicine, Caen University Hospital, University of Caen-Normandie, Ave de la Côte de Nacre, 14000 Caen, France; e-mail: deboysson-h@chu-caen.fr

-- Indicates open access to non-subscribers at www.ajnr.org

三 Indicates article with supplemental on-line table.

http://dx.doi.org/10.3174/ajnr.A5300
}

AJNR Am J Neuroradiol 38:1917-22 Oct 2017 www.ajnr.org 
diagnosis of PCNSV. Noncontrast 3D-TOF-MRA sequences can demonstrate vascular changes, such as stenosis, occlusions, or fusiform dilation. However, in many patients, DSA is still performed, even if the brain 3D-TOF-MRA shows vascular abnormalities. Our aim was to compare the diagnostic concordance between noncontrast intracranial 3D-TOF-MRA and DSA in patients with PCNSV.

\section{MATERIALS AND METHODS}

This study was conducted in accordance with Good Clinical Practices and the Declaration of Helsinki. Inclusion in the cohort required patient informed consent, and the cohort was approved by the Paris-Cochin institutional review board (No. 12541).

The article was prepared in accordance with Strengthening the Reporting of Observational Studies in Epidemiology ${ }^{8}$ and Standards for Reporting of Diagnostic Accuracy Studies (http://www. stard-statement.org/) guidelines.

\section{Patients}

We created a cohort of patients with definite diagnoses of PCNSV (COhort of patients with primary VAsculitis of the Central Nervous System [COVAC]) in 2010 in France using the well-established networks of the French Vasculitis Study Group, French NeuroVascular Society, and National Society of Internal Medicine. This cohort was supported by an institutional grant from the French Ministry of Health (COVAC, 2009 hospital project of clinical research [PHRC] 08017).

After the initial description of the first 52 enrolled patients, ${ }^{9}$ the cohort increased to 85 patients at the time of the present study.

As previously mentioned, patients were older than 18 years of age at diagnosis and had evidence of cerebral vascular involvement on brain biopsy and/or CNS vessel imaging. They had all been followed up for at least 6 months after diagnosis (unless they died) and were thoroughly screened at the onset for any differential diagnosis. We excluded patients with other vasculopathies or conditions potentially associated with secondary CNS vasculitis (eg, systemic vasculitis, autoimmune disease, infection). RCVS was excluded for each patient on the basis of the following criteria: 1) clinical presentation (no severe or thunderclap headaches at presentation, unless a brain biopsy showed vasculitis), 2) persistence of some vascular lesions as defined on MRA performed $>6$ months after the initial diagnostic work-up, and 3) clinical follow-up.

For the present study, we retrospectively selected in the COVAC population patients who underwent baseline intracranial 3D-TOFMRA and DSA before treatment initiation and within a 2-week interval. We did not include patients with both procedures having negative findings and negative or absent biopsy findings.

\section{Study Variables and Definitions}

For each patient, as previously described, ${ }^{9}$ we collected standard demographic data, medical history, clinical symptoms at disease onset, laboratory test parameters, CSF results (considered abnormal when the leukocyte count was $>5$ cells $/ \mathrm{mL}$ and/or the total protein level was $>0.5 \mathrm{mg} / \mathrm{mL}$ ), neuroimaging findings, and CNS histology, when available. Transmural inflammation of the vessel wall on a biopsy sample, with or without lymphocytic infiltrate, granuloma, and necrosis, defined biopsy-proved PCNSV. ${ }^{10}$

\section{Neuroimaging Analysis}

For each patient, all imaging examinations, including source images, were gathered in a digital anonymous format and forwarded for review by 2 neuroradiologists (with 14 [O.N.] and 6 [G.B.] years of experience in stroke imaging) who were blinded to clinical manifestations. Characteristics of each MR imaging unit were also collected. Proximal, second divisions, and subsequent branches of each cerebral artery and the vertebral artery, PICA, anterior inferior cerebellar artery, superior cerebellar artery (SCA), and basilar artery were analyzed with both procedures. For DSA and 3D-TOF-MRA analysis, in line with previous works, we differentiated large-, medium-, and small-sized vessels. ${ }^{1,12}$ Intracranial internal carotid and proximal anterior (A1), middle (M1), and posterior $(\mathrm{P} 1)$ cerebral arteries were considered large; second divisions (A2, M2, P2) and subsequent branches ( $>$ A2, $>$ M2, $>$ P2) were considered medium- and small-sized vessels, respectively. The vertebral and basilar arteries were considered largesized vessels, whereas the PICA, anterior inferior cerebellar artery, and SCA were considered medium-sized vessels. We thus divided cerebral vasculature into 25 segments (left and right intracranial internal carotid arteries, A1, A2, >A2, M1, M2, >M2, P1, P2, $>\mathrm{P} 2$; vertebral artery; and PICA/anterior inferior cerebellar artery/SCA, and basilar artery). A vascular segment was considered involved in case of stenosis, fusiform dilation, or occlusion.

We thus analyzed concordance of 1.5T/3T 3D-TOF-MRA and DSA.

\section{Statistical Analyses}

Categoric variables are expressed as number (\%), and quantitative variables, as median (range). Categoric variables were analyzed with the $\chi^{2}$ test, and quantitative variables were analyzed with the Wilcoxon rank sum test.

The Cohen $\kappa$ Concordance Index was used to analyze concordance between 3D-TOF-MRA and DSA in the global cerebral vasculature and in each vascular territory. Interrater agreement in identifying intracranial artery stenosis on DSA and 3D-TOFMRA was studied with the Cohen $\kappa$ Concordance Index. Any discordance between readers was adjudicated by consensus. The index values represent the following interpretations: poor $(\kappa<$ $0)$, slight $(\kappa=0-0.20)$, fair $(\kappa=0.21-0.40)$, moderate $(\kappa=$ $0.41-0.60)$, substantial $(\kappa=0.61-0.80)$, and almost perfect $(\kappa=$ 0.81-1).

Using DSA as a criterion standard, we calculated the sensitivity, specificity, accuracy, and positive and negative predictive values of MRA on per-patient and per-segment analyses. Statistical analyses were computed with JMP, Version 9.0.1, 2010 (SAS Institute, Cary, North Carolina), with $P<.05$ defining statistical significance.

\section{RESULTS}

Among the 85 patients included in COVAC, 31 (from 12 different centers) met the inclusion criteria and underwent both 3D-TOFMRA and DSA at baseline. The demographic data, clinical manifestations, and main laboratory test results of the 31 enrolled patients are shown in Table 1 . The other 54 patients were not included because they did not undergo both procedures before treatment $(n=21)$ or because of a lack of digitalized images to 
Table 1: Characteristics at diagnosis of the 31 patients with primary central nervous system vasculitis $^{\mathrm{a}}$

\begin{tabular}{lccc}
\hline \multicolumn{1}{c}{ Characteristics } & Total $(\boldsymbol{n}=\mathbf{3 1})$ & $\begin{array}{c}\text { Unenrolled } \\
\text { Patients }(\boldsymbol{n}=\mathbf{5 4})\end{array}$ & $\boldsymbol{P}$ Value \\
\hline Demographic data & & & \\
Age (yr) & $42(24-65)$ & $46(18-79)$ & .73 \\
Male/female & $16: 15$ & $28: 26$ & .98 \\
Clinical manifestations & & & \\
Headaches & $17(55)$ & $42(78)$ & .03 \\
Focal deficits & $26(84)$ & $43(80)$ & .63 \\
Seizures & $9(29)$ & $22(41)$ & .28 \\
Cognitive impairment & $7(23)$ & $26(48)$ & .02 \\
Loss of consciousness & $3(10)$ & $19(35)$ & .01 \\
Psychiatric disorders & $6(19)$ & $13(24)$ & .61 \\
C-reactive protein, (mg/L) & $3(0-47)$ & $5(1-95)$ & .13 \\
Abnormal CSF & $18 / 29(62)$ & $35 / 53(66)$ & .13 \\
Leukocyte count $\left(/ \mathrm{mm}{ }^{3}\right)$ & $6(1-100)$ & $8(0-250)$ & .07 \\
Protein concentration (mg/mL) & $0.6(0.2-4.1)$ & $0.7(0.1-2.8)$ & .19 \\
Abnormal brain biopsy findings & $8 / 16(50)$ & $18 / 26(69)$ & .32 \\
Abnormal DSA findings & $25(81)$ & $26 / 41(63)$ & .13 \\
Abnormal MRA findings & $24(77)$ & $25 / 44(57)$ & .09 \\
\hline
\end{tabular}

${ }^{a}$ Unless otherwise indicated, values are an absolute number (\%) or median (range).

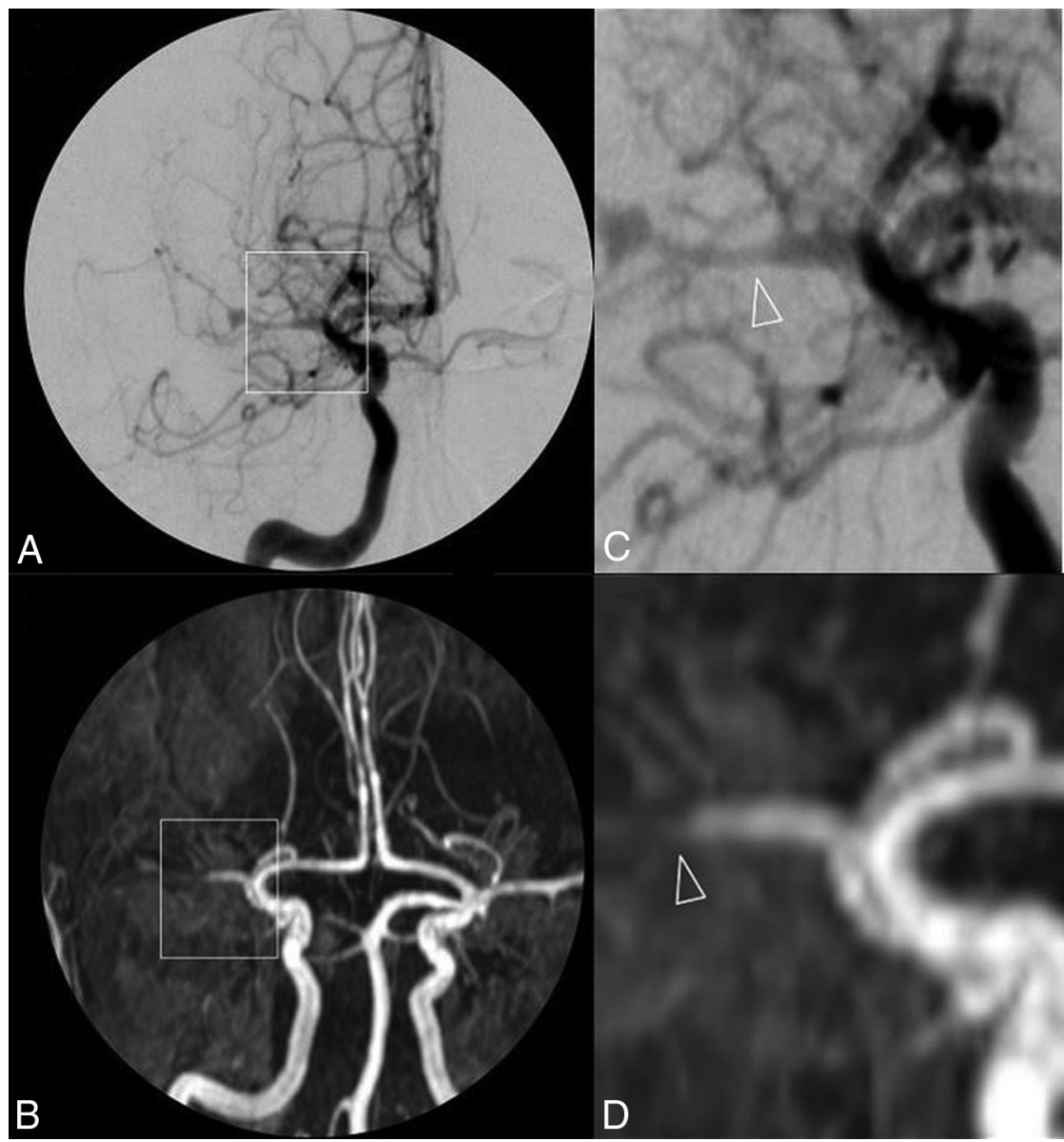

FIG 1. DSA $(A)$ and 3D-TOF-MRA $(C)$ with respective magnifications $(B$ and $D)$ of a patient with primary central nervous system vasculitis. A near-occlusive stenotic lesion of the proximal middle cerebral artery segment extending to both superficial branches with a pseudodilation of the bifurcation is shown. Arterial lumen irregularities are also observed in A2 segments of both anterior cerebral arteries.

review $(n=33)$. These excluded patients had more headaches, cognitive impairment, and loss of consciousness than those enrolled. They were more often in the biopsy-proved PCNSV group, and their DSA and 3D-TOF-MRA findings were often less posi- tive compared with included patients, though the differences were not statistically significant. Excluded and enrolled patients did not differ in terms of other demographic data, laboratory tests, neuroimaging results, treatment, and outcome.

Sixteen $(52 \%)$ of the 31 patients analyzed in this study had a brain biopsy that showed vasculitis features in 8 cases (50\%). The 23 patients without histologic proof of vasculitis had DSA-diagnosed PCNSV and did not show any clinical or biologic signs compatible with another vessel-related disease, including RCVS, during a median follow-up of 43 months (range, 14-78 months).

Brain MRIs with T1-weighted, T2weighted, fluid-attenuated inversion recovery, T1-weighted with gadolinium injection, gradient-echo $\mathrm{T} 2{ }^{*}$-weighted, and diffusion-weighted imaging with apparent diffusion coefficient mapping as well as 3D-TOF-MRA sequences were obtained on 1.5T and 3T MR imaging units in 20 and 11 patients, respectively. Characteristics of each MR imaging unit and sequence parameters are shown in the On-line Table. Interrater concordance in identifying intracranial artery lesions on DSA and 3D-TOF-MRA was excellent $(\kappa=0.93 ; 95 \%$ CI, $0.89-$ 0.96 ; and $\kappa=0.87 ; 95 \%$ CI, $0.79-0.92$, respectively).

Twenty-five (81\%) patients had abnormal DSA findings, and 24 (77\%) of them also had abnormal 3D-TOF-MRA findings ( 2 patients are presented in Figs 1 and 2). The patient with abnormal DSA findings but normal 3D-TOFMRA findings had isolated small-vessel involvement, demonstrated by brain biopsy. The 6 patients with normal DSA findings (and normal 3D-TOF-MRA findings) had biopsy-proved PCNSV.

In a per-segment analysis, medians of 2 (range, $0-18$ ) and 3 (range, $0-18$ ) abnormal segments were observed on 3D-TOF-MRA and DSA $(P=.03)$, respectively. 3D-TOF-MRA identified 116 of $141(82.3 \%)$ abnormal segments as observed on DSA. The other 25 falsenegative segments observed only on DSA but not on 3DTOF-MRA were small-sized vessels in 16 cases, mediumsized vessels in 8 cases, and large-sized vessels in 1 case. Seven false-positive vascular segments were involved in MRA but not 


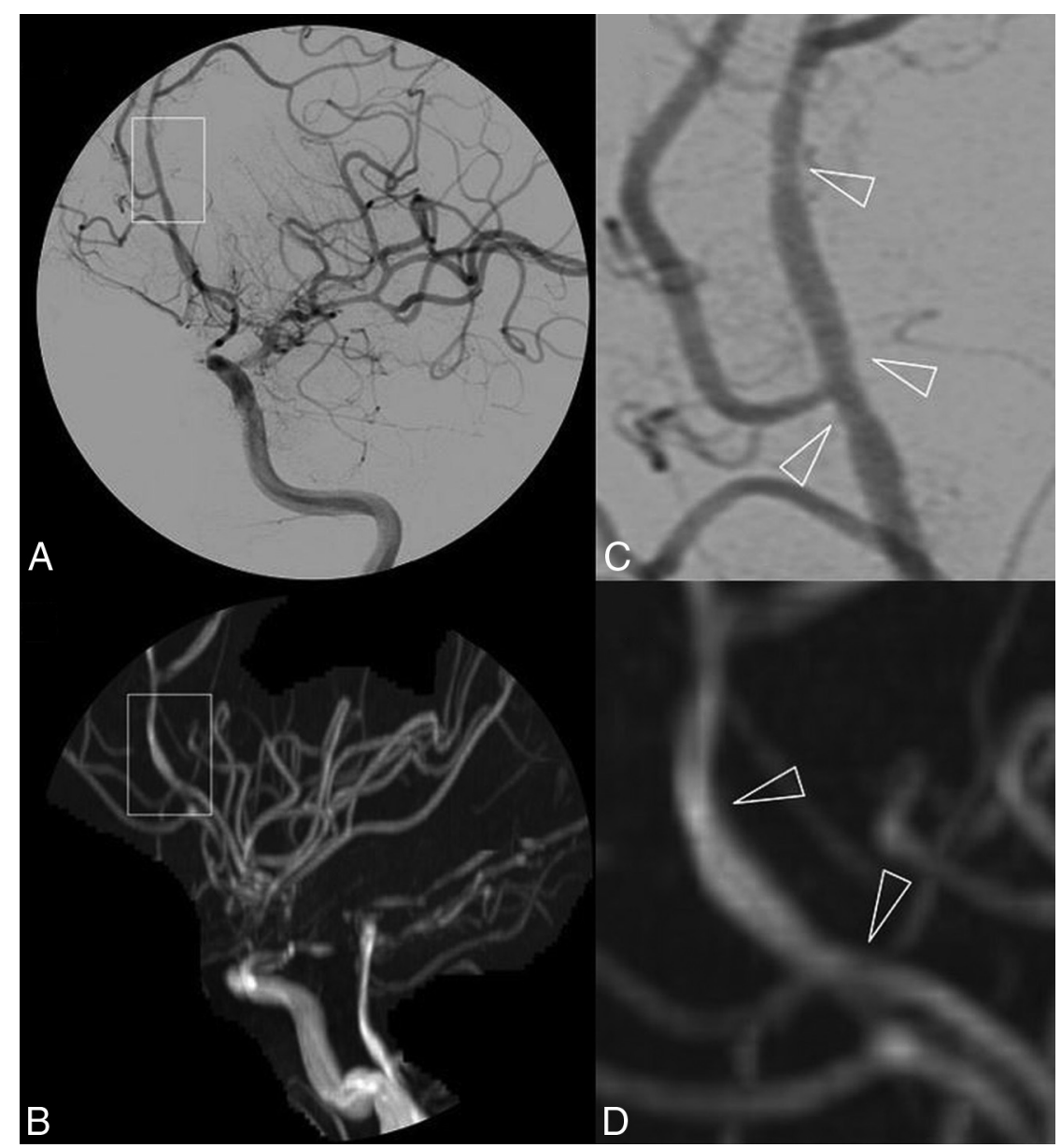

FIG 2. DSA $(A)$ and 3D-TOF-MRA $(C)$ with respective magnifications $(B$ and $D)$ of a patient with primary central nervous system vasculitis. Subtle irregularities of the arterial lumen of the $A 2-A 3$ segments of the left anterior cerebral artery are shown in a patient with otherwise near-occlusive stenoses of left intracranial internal carotid artery extending to the initial portion of the left MCA. was 0.87 (95\% CI, 0.78-0.91). Detailed $\kappa$ indices for each vascular segment according to the MR imaging unit are shown in Table 2.

All $\kappa$ indices were $>0.50$, even for small-sized vessels. When we assessed small-vessel involvement, 3T 3D-TOFMRA showed better results than $1.5 \mathrm{~T}$ 3D-TOF-MRA.

The sensitivity, specificity, diagnostic accuracy, and positive and negative predictive values of all MRAs, 1.5T MRAs, and 3T MRAs, with DSA as the criterion standard, are shown in Table 3.

\section{DISCUSSION}

Our results indicate excellent concordance between noncontrast 3D-TOFMRA and DSA in patients with PCNSV during the initial imaging work-up. For small-vessel involvement, 3T 3D-TOFMRA detected more distal vascular involvement than $1.5 \mathrm{~T}$ units.

In patients with suspected PCNSV, biopsy remains the best procedure to yield a diagnosis. In patients without or with negative biopsy findings, DSA is still considered the criterion standard for brain vessel imaging, but it is now performed less frequently than $3 \mathrm{D}$ TOF-MRA in many centers because of its more invasive nature. Typical DSA findings of PCNSV include alternating areas of stenosis and dilation, referred to
Table 2: Concordance between 1.5T/3T MRA and DSA on cerebral vascular segments in primary central nervous system vasculitis

\begin{tabular}{lcc}
\hline & $1.5 T$ MR $(\boldsymbol{\kappa} ; 95 \% \mathrm{CI})$ & 3T MR $(\boldsymbol{\kappa} ; 95 \% \mathrm{CI})$ \\
\hline $\begin{array}{l}\text { Intracranial internal } \\
\quad \text { carotid artery }\end{array}$ & 1 & 1 \\
$\mathrm{~A} 1$ & $0.90(0.73-1)$ & $0.90(0.75-1)$ \\
$\mathrm{A} 2$ & $0.83(0.59-1)$ & $0.83(0.51-1)$ \\
$>$ A2 & $0.57(0.34-0.72)$ & $0.86(0.60-1)$ \\
M1 & $0.94(0.81-1)$ & 1 \\
M2 & $0.90(0.76-1)$ & $0.91(0.82-1)$ \\
$>$ M2 & $0.69(0.41-0.97)$ & $0.74(0.42-1)$ \\
P1 & 1 & 1 \\
P2 & $0.60(0.20-0.98)$ & 1 \\
$>$ P2 & $0.64(0.18-1)$ & 1 \\
V4 & $0.66(0.38-1)$ & $0.86(0.62-1)$ \\
BA & $0.83(0.50-1)$ & 1 \\
PICA/AICA/SCA & $0.88(0.63-1)$ & 1 \\
\hline
\end{tabular}

Note:-BA indicates basilar artery.

on DSA: medium-sized vessels in 4 cases and small-sized vessels in 3 .

There was a high degree of concordance between 3D-TOFMRA and DSA ( $\kappa$ Index, 0.84; 95\% CI, 0.79-0.89; $P<.0001$ ). Concordance between 1.5T 3D-TOF-MRA and DSA was 0.82 (95\% CI, 0.75-0.93), and between 3T 3D-TOF-MRA and DSA, it as beading, which can be smooth or irregular. These typically occur bilaterally but can also include single vessels. Although DSA lesion patterns (in PCNSV) have been described in detail, ${ }^{6,7,13}$ no systematic comparison between 3D-TOF-MRA and DSA has been performed in PCNSV. In atherosclerosis or other vasculitis such as Takayasu arteritis, an excellent concordance was observed between MRA and DSA. ${ }^{14-18}$ Herein, 3D-TOF-MRA was able to demonstrate vascular involvement in different vessels with an efficiency similar to that of DSA. However, DSA showed a larger FOV compared with MRA and remains a better tool to give a precise and exhaustive cartography of vascular lesions, especially in the most distal vascular segments. Moreover, flow saturation lowers the detection of distal stenoses in 3D-TOF-MRA and thus may also explain the better ability of DSA to assess distal vascular segments. $^{19}$

As expected, compared with $1.5 \mathrm{~T}$ units, 3T 3D-TOF MRA showed better sensitivity and concordance with DSA. In smallsized vessel involvement, a high degree of concordance was observed between 3D-TOF-MRA and DSA only for 3T units. However, isolated small-sized vessel PCNSV involves a subgroup of patients in whom DSA findings (and a fortiori 3D-TOF-MRA) are also mostly negative, and only biopsy can show vascular involvement in these patients. ${ }^{9,20-22}$ In our first description of the French 
Table 3: Diagnostic performance of 1.5T and 3T 3D-TOF-MRA in primary central nervous system vasculitis compared with DSA

\begin{tabular}{lccccc}
\hline & Sensitivity (\%) & Specificity (\%) & Accuracy (\%) & PPV & NPV \\
\hline Diagnosis per patient & $96(24 / 25)$ & $100(6 / 6)$ & $96.77(30 / 31)$ & 100 & 85.7 \\
$\begin{array}{l}\text { Diagnosis per segment on } \\
\quad \text { all MR units }(n=31)\end{array}$ & $83.3(116 / 141)$ & $98.9(627 / 634)$ & $95.9(743 / 775)$ & 94.3 & 96.2 \\
1.5 MR units $(n=20)$ & $81.5(75 / 92)$ & $98.5(402 / 408)$ & $95.4(477 / 500)$ & 92.6 & 95.9 \\
3T MR units $(n=11)$ & $83.7(41 / 49)$ & $99.6(225 / 226)$ & $96.7(266 / 275)$ & 97.6 & 96.6 \\
\hline
\end{tabular}

Note:-PPV indicates positive predictive value; NPV, negative predictive value.

cohort, as well as in another large study by Salvarani et al, ${ }^{23}$ twothirds of the patients with biopsy-proved PCNSV had negative DSA findings. ${ }^{9}$ In the present study, 6 of our 7 patients with isolated small-sized vessel vasculitis demonstrated by biopsy had normal 3D-TOF-MRA and DSA findings. Hence, our results suggest that 3D-TOF-MRA could be performed as the first vascular imaging for patients with suspected PCNSV. When findings are negative, DSA could be the second imaging procedure, though our results showed that most patients would have negative DSA findings in this setting.

This study has limitations, including its retrospective design and relatively small sample size. Moreover, because this was a multicenter study, 3D-TOF-MRA was not performed with similar parameters in each center. The heterogeneity of MR imaging devices used at different sites and the use of $1.5 \mathrm{~T}$ or $3 \mathrm{~T}$ MR imaging are limitations because differences in acquisition parameters may influence both image quality and diagnosis. However, the demonstration of a good concordance between 1.5/3T 3D-TOF-MRA and DSA among manufacturers and MR imaging fields strengthens the conclusion that 3D-TOF-MRA can be used to demonstrate the involvement of multiple vessels in the working diagnosis of PCNSV. Regarding the visibility of distal branches on 3D-TOFMRA, the use of a multislab technique may have led to misinterpretation and false-positives for distal stenoses. However, distal stenoses observed on 3D-TOF-MRA were also observed on DSA, reducing the probability of false-positivity.

The patients analyzed in this study may represent a subset of those with PCNSV (selection bias) because we observed some differences in excluded patients, limiting the generalizability of our results. However, more cases of small-sized-vessel PCNSV were apparent among excluded patients - that is, biopsy-proved PCNSV with normal neurovascular imaging findings. Neuroradiologists who performed the neuroimaging analyses were aware that all patients in the series had PCNSV, which might also have introduced a bias in their interpretation of the images. Interpretation of multiple vascular narrowings or stenosis, including differentiation between PCNSV and distal intracranial atherosclerosis or RCVS, remains challenging. To minimize a false-positive diagnosis of PCNSV, we definitely included patients in the French PCNSV register after at least 6 months of follow-up to analyze their clinical and radiologic evolutions under treatment. This inclusion criteria retrospectively strengthened the confidence in the diagnosis. Because RCVS is the main differential in diagnosis of PCNSV in the case of multifocal segmental stenosis of cerebral arteries, a significant limitation of this study is that no patients had initial high-resolution black-blood contrast-enhanced 3D-T1WI of the intracranial arterial wall. In addition, some studies have suggested that arterial wall imaging may enable differentiation between RCVS and PCNSV with specific imaging patterns. ${ }^{24,25}$
Although the present study suggests that demonstration of multifocal segmental stenosis of cerebral arteries on 3D-TOF-MRA may be sufficient to strengthen the diagnostic suspicion of PCNSV, DSA may also be necessary to add precise lesional mapping in the distal vasculature and rule out mimics. Finally, we performed per-patient and per-segment analyses. In the latter, we may have artificially increased the diagnostic performance of MRA because readers classified each segment as normal or pathologic, irrespective of the number of focal stenoses.

\section{CONCLUSIONS}

The present study adds new insights to the diagnostic approach for patients with suspected PCNSV. 3D-TOF-MRA showed excellent concordance with DSA in demonstrating multiple vascular lesions. Higher performance was observed in distal vascular analysis with 3T 3D-TOF-MRA than with 1.5T units. Additionally, in patients with negative 3D-TOF-MRA findings, particularly obtained on a 3T MR imaging unit, the DSA yield was low. Further studies are required to determine whether 3T 3D-TOF-MRA can replace DSA.

\section{ACKNOWLEDGMENTS}

The authors thank Dr Alison Johnson for proof-reading the manuscript.

Disclosures: Emmanuel Touzé-UNRELATED: Board Membership: Boehringer Ingelheim; Payment for Lectures Including Service on Speakers Bureaus: Boehringer Ingelheim, Bristol-Myers Squibb, Amgen, Pfizer, Bayer; Travel/Accommodations/ Meeting Expenses Unrelated to Activities Listed: Teva Pharmaceutical Industries, Sanofi Genzyme. Mathieu Zuber-UNRELATED: Board Membership: Boehringer Ingelheim; Consultancy: Boehringer Ingelheim, Bayer; Payment for Lectures Including Service on Speakers Bureaus: Boehringer Ingelheim, Bayer, Sanofi. Christian Pagnoux-UNRELATED: Board Membership: Roche, Sanofi, ChemoCentryx, Comments: Less than $€ 15,000$ each during the past 5 years; Consultancy: Roche, Sanofi, ChemoCentryx, Comments: board and consultancy included, less than $€ 15,000$ each during the past 5 years; Grants/Grants Pending: Roche, Comments: educational in-aid grant $<15,000$ CAD in past 3 years; Payment for Lectures Including Service on Speakers Bureaus: Roche, Comments: Less than 5000 CAD during the past 3 years.

\section{REFERENCES}

1. Salvarani C, Brown RD Jr, Hunder GG. Adult primary central nervous system vasculitis. Lancet 2012;380:767-77 CrossRef Medline

2. Hajj-Ali RA, Calabrese LH. Central nervous system vasculitis. Curr Opin Rheumatol 2009;21:10-18 CrossRef Medline

3. Birnbaum J, Hellmann DB. Primary angiitis of the central nervous system. Arch Neurol 2009;66:704-09 Medline

4. Hajj-Ali RA, Singhal AB, Benseler S, et al. Primary angiitis of the CNS. Lancet Neurol 2011;10:561-72 CrossRef Medline

5. Calabrese LH, Mallek JA. Primary angiitis of the central nervous system: report of 8 new cases, review of the literature, and proposal for diagnostic criteria. Medicine (Baltimore) 1988;67:20-39 CrossRef Medline

6. Alhalabi M, Moore PM. Serial angiography in isolated angiitis of the central nervous system. Neurology 1994;44:1221-26 CrossRef Medline

7. Kadkhodayan Y, Alreshaid A, Moran CJ, et al. Primary angiitis of the central nervous system at conventional angiography. Radiology 2004;233:878-82 CrossRef Medline

8. von Elm E, Altman DG, Egger M, et al; STROBE Initiative. The Strengthening the Reporting of Observational Studies in Epidemi-

AJNR Am J Neuroradiol 38:1917-22 Oct 2017 www.ajnr.org

1921 
ology (STROBE) statement: guidelines for reporting observational studies. PLoS Med 2007;4:e296 CrossRef Medline

9. de Boysson H, Zuber M, Naggara O, et al; French Vasculitis Study Group and the French NeuroVascular Society. Primary angiitis of the central nervous system: description of the first fifty-two adults enrolled in the French cohort of patients with primary vasculitis of the central nervous system. Arthritis Rheumatol 2014;66:1315-26 CrossRef Medline

10. Miller DV, Salvarani C, Hunder GG, et al. Biopsy findings in primary angiitis of the central nervous system. Am J Surg Pathol 2009;33: 35-43 CrossRef Medline

11. MacLaren K, Gillespie J, Shrestha S, et al. Primary angiitis of the central nervous system: emerging variants. QJM 2005;98:643-54 CrossRef Medline

12. Salvarani C, Brown RD Jr, Calamia KT, et al. Primary central nervous system vasculitis: analysis of 101 patients. Ann Neurol 2007;62: 442-51 CrossRef Medline

13. Hellmann DB, Roubenoff R, Healy RA, et al. Central nervous system angiography: safety and predictors of a positive result in 125 consecutive patients evaluated for possible vasculitis. J Rheumatol 1992; 19:568-72 Medline

14. Choi CG, Lee DH, Lee JH, et al. Detection of intracranial atherosclerotic steno-occlusive disease with $3 \mathrm{D}$ time-of-flight magnetic resonance angiography with sensitivity encoding at 3T. AJNR Am J Neuroradiol 2007;28:439-46 Medline

15. Demaerel P, De Ruyter N, Maes F, et al. Magnetic resonance angiography in suspected cerebral vasculitis. Eur Radiol 2004;14:1005-12 CrossRef Medline

16. Garg SK, Mohan S, Kumar S. Diagnostic value of 3D contrast-enhanced magnetic resonance angiography in Takayasu's arteritis: a comparative study with digital subtraction angiography. Eur Radiol 2011;21:1658-66 CrossRef Medline

17. Korogi $\mathrm{Y}$, Takahashi $\mathrm{M}$, Nakagawa $\mathrm{T}$, et al. Intracranial vascular stenosis and occlusion: MR angiographic findings. AJNR Am J Neuroradiol 1997;18:135-43 Medline

18. Lee NJ, Chung MS, Jung SC, et al. Comparison of high-resolution MR imaging and digital subtraction angiography for the characterization and diagnosis of intracranial artery disease. $A J N R A m J N e u-$ roradiol 2016;37:2245-50 CrossRef Medline

19. Ozsarlak O, Van Goethem JW, Maes M, et al. MR angiography of the intracranial vessels: technical aspects and clinical applications. Neuroradiology 2004;46:955-72 CrossRef Medline

20. Benseler SM, deVeber G, Hawkins C, et al. Angiography-negative primary central nervous system vasculitis in children: a newly recognized inflammatory central nervous system disease. Arthritis Rheum 2005;52:2159-67 CrossRef Medline

21. Salvarani C, Brown RD Jr, Calamia KT, et al. Primary central nervous system vasculitis with prominent leptomeningeal enhancement: a subset with a benign outcome. Arthritis Rheum 2008;58:595-603 CrossRef Medline

22. de Boysson $\mathrm{H}$, Boulouis $\mathrm{G}$, Aouba A, et al. Adult primary angiitis of the central nervous system: isolated small-vessel vasculitis represents distinct disease pattern. Rheumatology (Oxford) 2017;56: 439-44 CrossRef Medline

23. Salvarani C, Brown RD Jr, Christianson TJ, et al. Adult primary central nervous system vasculitis treatment and course: analysis of one hundred sixty-three patients. Arthritis Rheumatol 2015;67:1637-45 CrossRef Medline

24. Mandel DM, Matouk CC, Farb RI, et al. Vessel wall MRI to differentiate between reversible cerebral vasoconstriction syndrome and central nervous system vasculitis: preliminary results. Stroke 2012; 43:860-62 CrossRef Medline

25. Obusez EC, Hui F, Hajj-Ali RA, et al. High-resolution MRI wall imaging: spatial and temporal patterns of reversible cerebral vasoconstriction syndrome and central nervous system vasculitis. AJNR Am J Neuroradiol 2014;35:1527-32 CrossRef Medline 